\title{
Comparison of clinicopathological characteristics between cirrhotic and non-cirrhotic patients with intrahepatic cholangiocarcinoma: A large-scale retrospective study
}

\author{
LEI YUAN $^{1 *}$, XIANWU LUO $^{1 *}$, XINYUAN LU $^{2}$, BIN YI ${ }^{1}$, KAIJIAN CHU ${ }^{1}$, QUANYU CAI ${ }^{3}$ and XIAOQING JIANG \\ ${ }^{1}$ The First Department of Biliary Surgery, Departments of ${ }^{2}$ Pathology and ${ }^{3}$ Medical Imaging, Eastern Hepatobiliary \\ Surgery Hospital, Second Military Medical University, Shanghai 200438, P.R. China
}

Received September 25, 2016; Accepted July 22, 2017

DOI: $10.3892 / \mathrm{mco} .2017 .1387$

\begin{abstract}
The effect of cirrhosis on the characteristics of intrahepatic cholangiocarcinoma (ICC) has not been fully elucidated. The purpose of this study was to investigate how cirrhosis affects the clinicopathological characteristics and survival of surgically treated ICC patients. A total of 1,312 ICC patients surgically treated between January 2007 and December 2011 at a single institution were retrospectively reviewed and the clinicopathological data were compared between cirrhotic and non-cirrhotic patients. Univariate and multivariate analyses were performed to identify significant and independent prognostic factors in this cohort. A total of 302 patients $(23.0 \%)$ were cirrhotic. Compared with cirrhotic patients, the tumors in non-cirrhotic patients were usually larger, less differentiated, and more likely to have lymphatic metastasis, vascular and perineural invasion. Following resection, cirrhotic patients achieved a longer survival compared with non-cirrhotic patients (16.0 vs. 13.0 months, respectively; $\mathrm{P}<0.038)$. Multivariate analysis demonstrated that hepatitis $B$ virus infection and cirrhosis were independent favorable prognostic factors, while the presence of cholelithiasis, elevated carbohydrate antigen 19-9 and carcinoembryonic antigen levels, multiple tumors, lymphatic metastasis, vascular
\end{abstract}

Correspondence to: Dr Xiaoqing Jiang, The First Department of Biliary Surgery, Eastern Hepatobiliary Surgery Hospital, Second Military Medical University, 225 Changhai Road, Shanghai 200438, P.R. China

E-mail: jiangxiaoqingpro@sina.com

Abbreviations: AFP, $\alpha$-fetoprotein; CA19-9, carbohydrate antigen 19-9; CEA, carcinoembryonic antigen; CT, computed tomography; HBV, hepatitis B virus; HCC, hepatocellular carcinoma; ICC, intrahepatic cholangiocarcinoma; LN, lymph node; MRI, magnetic resonance imaging; MST, median survival time; OS, overall survival

\section{${ }^{*}$ Contributed equally}

Key words: intrahepatic cholangiocarcinoma, cirrhosis, surgical resection, survival invasion and positive surgical margin status were independent unfavorable prognostic factors. Overall, the clinicopathological characteristics of ICC patients with and without cirrhosis differed significantly. Compared with cirrhotic patients, in whom the biological behavior of ICC was similar to that of HCC, non-cirrhotic patients exhibited higher-risk pathological characteristics, lower curative resection rate and worse survival.

\section{Introduction}

Intrahepatic cholangiocarcinoma (ICC) is the second most common primary liver cancer after hepatocellular carcinoma (HCC) (1-3). ICC has been categorized as peripheral and perihilar types based on location $(1,4)$, and as mass-forming, periductular infiltrating and intraductal growth types, based on the growth pattern classification of ICC by the Liver Cancer Study Group of Japan (5). An increasing number of studies suggest that surgical resection usually offers the possibility of long-term survival to patients with this disease $(2,6,7)$. Although there has been a worldwide increase in the incidence and mortality of ICC in recent years $(2,3)$, ICC has not been investigated as extensively as HCC (1).

Previous studies suggested that hepatitis B virus (HBV) infection and cirrhosis, which are well-documented pathogenic factors in the development of HCC (8-11), may also be associated with an increased risk of ICC (12-15). Cirrhosis is common among HCC patients (8-10), and has been proven to be a poor prognostic factor following surgical treatment of HCC $(10,16,17)$. A significant proportion of ICC patients are also cirrhotic; however, the prognostic role of this finding has not been extensively investigated. Although HBV infection has been reported to be a favorable prognostic factor for ICC patients and the clinicopathological characteristics differ between patients with and those without HBV infection $(13,18,19)$, the role of cirrhosis in the prognosis of ICC patients has not been fully elucidated due to the limited number of related studies. Cirrhosis has been found to be a favorable prognostic factor for ICC patients in our former study (20); however, the opposite result was reported by another previous study (21). The aim of the present study was to determine the effect of cirrhosis on the prognosis of ICC patients and the 
mechanism underlying this effect through comparing clinicopathological characteristics and survival data in large series of ICC patients with and without cirrhosis.

\section{Patients and methods}

Patients recruiting and grouping. A retrospective study was undertaken, including all consecutive patients with ICC who were admitted to the Eastern Hepatobiliary Surgery Hospital (Shanghai, China) for initial surgical treatment between January 2007 and December 2011. The inclusion criteria were as follows: No history of previous anticancer therapy and no history of other malignancies; no severe comorbidities that may affect survival; potentially resectable ICC on preoperative imaging; and no general contraindications to surgery. The exclusion criteria were as follows: Hilar or extrahepatic cholangiocarcinoma; combined HCC and cholangiocarcinoma; periductular infiltrating type and intraductal growth pattern of ICC; Child's C liver function; hepatitis $\mathrm{C}$ virus infection; definitive distant metastasis beyond the abdomen; and incomplete survival data. The patients were identified through computerized hospital databases. Subsequently, demographic data were collected for each patient, including age, gender, symptoms, underlying liver diseases, imaging findings, laboratory tests and pathological results. The patients were divided into two groups according to the presence or absence of cirrhosis, which was defined as widespread disruption of normal liver structure by the formation of pseudolobules or Scheuer stage 4 fibrosis in pathological findings (22). The protocol of the present study was approved by the local Ethics Committee.

Preoperative workup. The preoperative workup included abdominal ultrasonography, computed tomography (CT) and/or magnetic resonance imaging (MRI), cardiac and pulmonary function testing, endoscopic examination and laboratory tests. For patients with local or complete biliary obstruction, endoscopic retrograde cholangiopancreatography or magnetic resonance cholangiopancreatography was performed. In patients with suspected metastasis, positron emission tomography (PET) and CT (PET-CT) was performed.

Surgery. Patients underwent R0 (curative) or R1 (microscopic infiltration of the resection margin) liver resection, apart from cases where distant metastases, peritoneal carcinomatosis, extensive vascular involvement and/or multiple intrahepatic metastases were identified intraoperatively. R2 (palliative) resection and exploratory laparotomy with biopsy intention prior to surgery were not recommended, except when the abovementioned unfavorable findings during intraoperative exploration were beyond the preoperative evaluation of ICC and R0/R1 resection could not be performed. The majority of the liver resections were performed under vascular control, and anatomical or non-anatomical hepatectomy was determined depending on the size and location of tumor, as well as on the background of chronic liver disease. The types of hepatic resection performed included segmentectomy or local resection, bisegmentectomy, right or left hemihepatectomy, and extended hemihepatecomy, according to the 2000 Brisbane Classification of the International Hepato-Pancreato-Biliary Association (23). Additional procedures included cholecystectomy, resection of the biliary confluence and extrahepatic bile duct with Roux-en-Y hepatojejunostomy, portal vein cancerous thrombectomy, and vascular reconstruction. In patients with R0/R1 liver resection and suspected lymph node (LN) metastasis, LN dissection was performed when possible. In other patients with R0/R1 tumor resection and without evidence of macroscopic $\mathrm{LN}$ enlargement, preventive skeletonization of the hepatoduodenal ligament was performed to confirm the stage.

Pathological and immunohistochemical methods. All the resected and bioptic specimens were pathologically examined, including tumor size and number, capsule formation, LN metastasis, vascular invasion, perineural invasion and tumor cell differentiation. Each tumor was staged according to the 7th edition of the American Joint Committee on Cancer (AJCC) staging system for ICC (24). The surgical margins were examined for the presence of residual tumor and were classified according to the $\mathrm{R}$ classification as $\mathrm{R} 0$ (no residual tumor and resection margin $>0 \mathrm{~mm}$ ), $\mathrm{R} 1$ (microscopic residual tumor or null-margin resection) or R2 (macroscopic residual tumor) (25). Curative resection was defined a negative resection margin on histopathological examination.

Follow-up. All the patients were followed up postoperatively by X-ray of the chest, ultrasound scan of the liver, liver function tests and serum levels of carbohydrate antigen (CA) 19-9, carcinoembryonic antigen (CEA) and $\alpha$-fetoprotein (AFP) at an interval of 1-3 months. When recurrence or metastasis were suspected, a CT or MRI scan was performed to confirm the diagnosis. Treatments for recurrent disease included surgery, transarterial chemoembolization, radiotherapy and supportive therapy. Survival was evaluated from the date of surgery; the patients were followed up for survival until death or until the study deadline date of September 30, 2014.

Statistical analysis. Continuous variables are presented as the mean \pm standard deviation or as median values and range. Categorical variables are presented as total and percentage. Comparisons were performed using the unpaired t-test for continuous variables and the Chi-squared or Wilcoxon test for categorical variables. Overall survival (OS) rates were calculated using the Kaplan-Meier method. The statistically significant prognostic factors were analyzed by univariate analysis, evaluated using the Kaplan-Meier method and compared with the log-rank test. The multivariate analysis was performed using the Cox proportional hazards model to identify the independent prognostic factors for survival. Statistical analysis was performed using the SPSS 19.0 software for Windows (SPSS Inc., Chicago, IL). Differences with P-values of $<0.05$ were considered statistically significant.

\section{Results}

Clinical characteristics. A total of 1,312 patients with ICC were recruited, with a male predominance (896 patients; $68.3 \%$ ) and a median age of 54 years (range, $18-82$ years). The patients included $302(23.0 \%)$ with and 1,010 (77.0\%) without cirrhosis. The differences in clinical characteristics between the two groups of patients are listed in Table I. Compared with patients without cirrhosis, those with cirrhosis were younger, 
Table I. Comparison of clinical characteristics between intrahepatic cholangiocarcinoma patients with and without cirrhosis.

\begin{tabular}{|c|c|c|c|}
\hline Characteristics & $\begin{array}{l}\text { With cirrhosis, } n(\%) \\
\qquad(\mathrm{n}=302)\end{array}$ & $\begin{array}{l}\text { Without cirrhosis, } \\
\mathrm{n}(\%)(\mathrm{n}=1,010)\end{array}$ & P-value \\
\hline Age (years) & & & $<0.001$ \\
\hline Mean \pm standard deviation & $51.65 \pm 10.05$ & $54.80 \pm 11.07$ & \\
\hline Gender & & & $<0.001$ \\
\hline Male & $270(89.4)$ & $626(62.0)$ & \\
\hline Female & $32(10.6)$ & $384(38.0)$ & \\
\hline Symptoms & & & $<0.001$ \\
\hline No & $155(51.3)$ & $344(34.1)$ & \\
\hline Yes & $147(48.7)$ & $666(65.9)$ & \\
\hline HBsAg positivity & $272(90.1)$ & $326(32.3)$ & $<0.001$ \\
\hline Alcoholic & $59(19.5)$ & $131(13.0)$ & 0.004 \\
\hline Schistosomiasis & $7(2.3)$ & $64(6.3)$ & 0.007 \\
\hline Cholelithiasis & $30(9.9)$ & $224(22.2)$ & $<0.001$ \\
\hline Elevated AFP level & $119(39.4)$ & $129(12.8)$ & $<0.001$ \\
\hline Elevated CA19-9 and/or CEA level & $143(47.4)$ & $633(62.7)$ & $<0.001$ \\
\hline Albumin (g/l) & & & 0.164 \\
\hline Mean \pm standard deviation & $41.53 \pm 3.97$ & $41.91 \pm 4.29$ & \\
\hline Bilirubin $(\mu \mathrm{mol} / \mathrm{l})$ & & & 0.016 \\
\hline$\leq 20$ & $243(80.5)$ & $870(86.1)$ & \\
\hline$>20$ & $59(19.5)$ & $140(13.9)$ & \\
\hline ALT(U/1) & & & 0.001 \\
\hline$\leq 42$ & $197(65.2)$ & 759 (75.1) & \\
\hline$>42$ & $105(34.8)$ & $251(24.9)$ & \\
\hline
\end{tabular}

AFP, $\alpha$-fetoprotein; CA19-9, carbohydrate antigen 19-9; CEA, carcinoembryonic antigen; HBsAg, hepatitis B surface antigen; ALT, alanine aminotransferase.

included a higher percentage of men, and fewer had symptoms, elevated serum levels of CA19-9 and/or CEA or other concurrent liver diseases, such as schistosomiasis and cholelithiasis; however, a higher percentage of cirrhotic patients had elevated serum levels of AFP.

Pathological characteristics. On pathological examination, more patients without cirrhosis had well/moderately differentiated tumors, while more patients with cirrhosis had tumors with capsule formation (Table II). Patients with cirrhosis had relatively smaller tumors, with a lower likelihood of LN metastasis and perineural invasion, but with a higher likelihood of vascular invasion when compared with patients without cirrhosis. According to the 7th edition of the AJCC staging system, 506 cases $(38.6 \%)$ were stage I, $323(24.5 \%)$ were stage II, 103 (7.9\%) were stage III and 380 (29.0\%) were stage IV; more patients with cirrhosis had tumors at an earlier stage compared with those without cirrhosis (Table II).

Surgical results. Of the 1,312 ICC patients undergoing surgery, 1,260 received tumor resection (overall resectability rate, 96.0\%), among whom 296 (98.0\%) were cirrhotic and $964(95.4 \%)$ were non-cirrhotic. The types of liver resection included extended right or left hemihepatectomy in 69 (5.3\%), right or left hemihepatectomy in 424 (32.3\%), bisegmentectomy in $517(39.4 \%)$, and segmentectomy or local resection in 250 $(19.1 \%)$ patients; in patients with multiple tumors, different types of liver resections were used in combination, depending on the location and number of the tumors. The distribution of different types of liver resection in patients with and without cirrhosis is shown in Table III. Compared with cirrhotic patients, a wider resection range was more common among non-cirrhotic patients.

$\mathrm{R} 0, \mathrm{R} 1$ and $\mathrm{R} 2$ resection was performed in 454 (34.6\%), 591 $(45.0 \%)$ and $215(16.4 \%)$ patients, respectively; the remaining $52(4.0 \%)$ patients only underwent exploratory laparotomy with biopsy due to unresectable disease (e.g., extensive intrahepatic metastases or peritoneal seeding). Compared with patients without cirrhosis, a significantly higher rate of $\mathrm{R} 0$ resection was achieved in patients with cirrhosis (Table III).

Survival of the entire cohort. The duration of survival was defined as the time from surgery to the date of death or the last follow-up, and the median follow-up period was 47 months (range, 1-93 months). The 1-, 3- and 5-year OS rates for the entire cohort were 57.0, 19.9 and $13.3 \%$, respectively, with a median survival time (MST) of 14.0 months.

Survival of patients with and without cirrhosis. A significant difference in survival rates was observed between patients 
Table II. Comparison of pathological characteristics between intrahepatic cholangiocarcinoma patients with and without cirrhosis.

\begin{tabular}{lcc}
\hline Characteristics & $\begin{array}{c}\text { With cirrhosis, }(\%) \\
(\mathrm{n}=302)\end{array}$ & $\begin{array}{c}\text { Without cirrhosis, } \mathrm{n}(\%) \\
(\mathrm{n}=1,010)\end{array}$ \\
\hline Tumor size $(\mathrm{cm})$ & & $7.29 \pm 3.53$ \\
Mean \pm standard deviation & $6.39 \pm 3.69$ & \\
Tumor number & & $637(63.1)$ \\
Single & $202(66.9)$ & $373(36.9)$ \\
Multiple & $100(33.1)$ & $36(3.6)$ \\
Capsule formation & $40(13.2)$ & $944(93.5)$ \\
Differentiation & & $66(6.5)$ \\
High or moderate & $269(89.1)$ & $318(31.5)$ \\
Poor & $33(10.9)$ & $111(11.0)$ \\
Lymphatic metastasis & $52(17.2)$ & $91(9.0)$ \\
Vascular invasion & $92(30.5)$ & $<.001$ \\
Perineural invasion & $5(1.7)$ & $604(59.8)$ \\
Stage & & $406(40.2)$
\end{tabular}

Table III. Distribution of different types of liver resection in intrahepatic cholangiocarcinoma patients with and without cirrhosis.

\begin{tabular}{|c|c|c|c|}
\hline Types of liver resection & With cirrhosis, $\mathrm{n}(\%)$ & Without cirrhosis, $\mathrm{n}(\%)$ & P-value \\
\hline Surgical range & 296 & 964 & \\
\hline Segmentectomy or local resection & $90(30.4)$ & $160(16.6)$ & $<0.001$ \\
\hline Bisegmentectomy & $129(43.6)$ & $388(40.2)$ & 0.308 \\
\hline Hemihepatectomy & $63(21.3)$ & $361(37.4)$ & $<0.001$ \\
\hline Extended hemihepatectomy & $14(4.7)$ & $55(5.7)$ & 0.519 \\
\hline Surgical margin status & 302 & 1,010 & \\
\hline $\mathrm{R} 0$ resection & $146(48.3)$ & $308(30.5)$ & $<0.001$ \\
\hline $\mathrm{R} 1$ resection & $113(37.4)$ & $478(47.3)$ & 0.002 \\
\hline $\mathrm{R} 2$ resection & $37(12.3)$ & $178(17.6)$ & 0.027 \\
\hline Exploratory laparotomy & $6(2.0)$ & $46(4.6)$ & 0.045 \\
\hline
\end{tabular}

with and those without cirrhosis; the 1-, 3- and 5-year OS rates for patients with and without cirrhosis were 62.3, 24.1 and 14.7\% (MST, 16.0 months), and 55.4, 18.7 and 13.0\% (MST, 13.0 months), respectively ( $\mathrm{P}<0.038$, Fig. 1$)$.

Univariate and multivariate analyses. The univariate analysis demonstrated that certain variables, including HBV infection, cirrhosis, presence of cholelithiasis, serum level of CA19-9 and/or CEA, tumor size, tumor number, capsule formation, LN metastasis, vascular invasion, perineural invasion and surgical margin status, were statistically significant prognostic factors affecting the survival of ICC patients (Table IV). Cox's regression multivariate analysis identified $\mathrm{HBV}$ infection and cirrhosis as independent favorable prognostic factors, while the presence of cholelithiasis, elevated CA19-9 and CEA levels, multiple tumors, lymphatic metastasis, vascular invasion and positive surgical margin status were independent unfavorable prognostic factors, with hazard ratios of 1.330, 1.726, 1.380, 1.297, 1.193 and 1.788, respectively (Table IV).

\section{Discussion}

ICC is a heterogeneous group of tumors, with different risk factors, biological behavior and clinicopathological characteristics and, consequently, different prognosis $(1,2,6,15)$. $\mathrm{HBV}$ infection and cirrhosis are established risk factors for HCC $(8,11)$, and several recent studies have suggested HBV infection may also be associated with the occurrence of ICC $(12,13,18,19)$; however, the association between cirrhosis and the pathogenesis/prognosis of ICC remains unknown. The present study confirmed the earlier observation that cirrhosis is prevalent among patients with ICC in highly endemic areas (6), as it was observed in $23.0 \%$ of our patients, which is a markedly higher percentage compared with Western 
Table IV. Univariate and multivariate analyses of variables associated with overall survival after surgery in 1,312 patients with intrahepatic cholangiocarcinoma.

\begin{tabular}{|c|c|c|c|c|c|}
\hline \multirow[b]{2}{*}{ Variables } & \multirow[b]{2}{*}{$\mathrm{N}(\%)$} & \multirow{2}{*}{$\begin{array}{l}\text { Median } \\
\text { survival } \\
\text { (months) }\end{array}$} & \multirow{2}{*}{$\begin{array}{l}\text { Univariate } \\
\text { analysis } \\
\text { (P-value) }\end{array}$} & \multicolumn{2}{|c|}{ Multivariate analysis } \\
\hline & & & & P-value & $\mathrm{HR}(95 \% \mathrm{CI})$ \\
\hline Age (years) & & & 0.795 & - & - \\
\hline$\leq 60$ & $920(70.1)$ & 14 & & & \\
\hline$>60$ & $392(29.9)$ & 14 & & & \\
\hline Gender & & & 0.736 & - & - \\
\hline Male & $896(68.3)$ & 14 & & & \\
\hline Female & $416(31.7)$ & 13 & & & \\
\hline $\mathrm{HBsAg}$ & & & $<0.001$ & $<0.001$ & $1.435(1.248-1.649)$ \\
\hline$(-)$ & $714(54.4)$ & 12 & & & \\
\hline$(+)$ & $598(45.6)$ & 19 & & & \\
\hline Cirrhosis & & & 0.038 & $<0.001$ & $1.367(1.161-1.609)$ \\
\hline No & $1,010(77.0)$ & 13 & & & \\
\hline Yes & $302(23.0)$ & 16 & & & \\
\hline Alcoholic & & & 0.473 & - & \\
\hline No & $1,122(85.5)$ & 14 & & & \\
\hline Yes & $190(14.5)$ & 15 & & & \\
\hline Schistosomiasis & & & 0.762 & - & \\
\hline No & $1,241(94.6)$ & 14 & & & \\
\hline Yes & $71(5.4)$ & 13 & & & \\
\hline Cholelithiasis & & & $<0.001$ & $<0.001$ & $1.330(1.145-1.545)$ \\
\hline No & $1,058(80.6)$ & 15 & & & \\
\hline Yes & 254 (19.4) & 10 & & & \\
\hline AFP elevation & & & 0.675 & & \\
\hline No & $1,064(81.1)$ & 14 & & & \\
\hline Yes & $248(19.0)$ & 14 & & & \\
\hline $\begin{array}{l}\text { CA19-9 and/or CEA } \\
\text { elevation }\end{array}$ & & & $<0.001$ & $<0.001$ & $1.726(1.520-1.959)$ \\
\hline No & $536(40.9)$ & 23 & & & \\
\hline Yes & $776(59.1)$ & 11 & & & \\
\hline Tumor size $(\mathrm{cm})$ & & & $<0.001$ & 0.105 & - \\
\hline$\leq 5$ & $464(35.4)$ & 20 & & & \\
\hline$>5$ & $848(64.6)$ & 12 & & & \\
\hline Tumor number & & & $<0.001$ & $<0.001$ & $1.380(1.212-1.571)$ \\
\hline Single & 839 (63.9) & 18 & & & \\
\hline Multiple & $473(36.1)$ & 10 & & & \\
\hline Capsule formation & & & $<0.001$ & 0.141 & - \\
\hline No & $1,236(94.2)$ & 14 & & & \\
\hline Yes & $76(5.8)$ & 25 & & & \\
\hline Lymph node metastasis & & & $<0.001$ & 0.001 & $1.297(1.110-1.515)$ \\
\hline No & $942(71.8)$ & 18 & & & \\
\hline Yes & $370(28.2)$ & 8 & & & \\
\hline Vascular invasion & & & 0.010 & 0.036 & $1.193(1.012-1.407)$ \\
\hline No & $1,109(84.5)$ & 14 & & & \\
\hline Yes & $203(15.5)$ & 13 & & & \\
\hline Perineural invasion & & & 0.001 & 0.235 & - \\
\hline No & $1,216(92.7)$ & 14 & & & \\
\hline Yes & $96(7.3)$ & 11 & & & \\
\hline Differentiation & & & 0.836 & - & - \\
\hline High or moderate & $1,213(92.5)$ & 14 & & & \\
\hline Poor & $99(7.5)$ & 13 & & & \\
\hline
\end{tabular}


Table IV. Continued.

\begin{tabular}{|c|c|c|c|c|c|}
\hline Variables & $\mathrm{N}(\%)$ & $\begin{array}{l}\text { Median } \\
\text { survival } \\
\text { (months) }\end{array}$ & $\begin{array}{c}\text { Univariate } \\
\text { analysis } \\
\text { (P-value) }\end{array}$ & $\begin{array}{c}\text { Multivariate } \\
\text { analysis } \\
\text { P-value }\end{array}$ & $\mathrm{HR}(95 \% \mathrm{CI})$ \\
\hline Surgical margin status & & & $<0.001$ & $<0.001$ & $1.788(1.612-1.984)$ \\
\hline $\mathrm{R} 0$ resection & $454(34.6)$ & 24 & & & \\
\hline $\mathrm{R} 1$ resection & $591(45.0)$ & 14 & & & \\
\hline $\mathrm{R} 2$ resection & $215(16.4)$ & 6 & & & \\
\hline Exploratory laparotomy & $52(4.0)$ & 4 & & & \\
\hline
\end{tabular}

AFP, $\alpha$-fetoprotein; HBsAg, hepatitis B surface antigen; CA19-9, carbohydrate antigen 19-9; CEA, carcinoembryonic antigen; HR, hazard ratio; $\mathrm{CI}$, confidence interval.

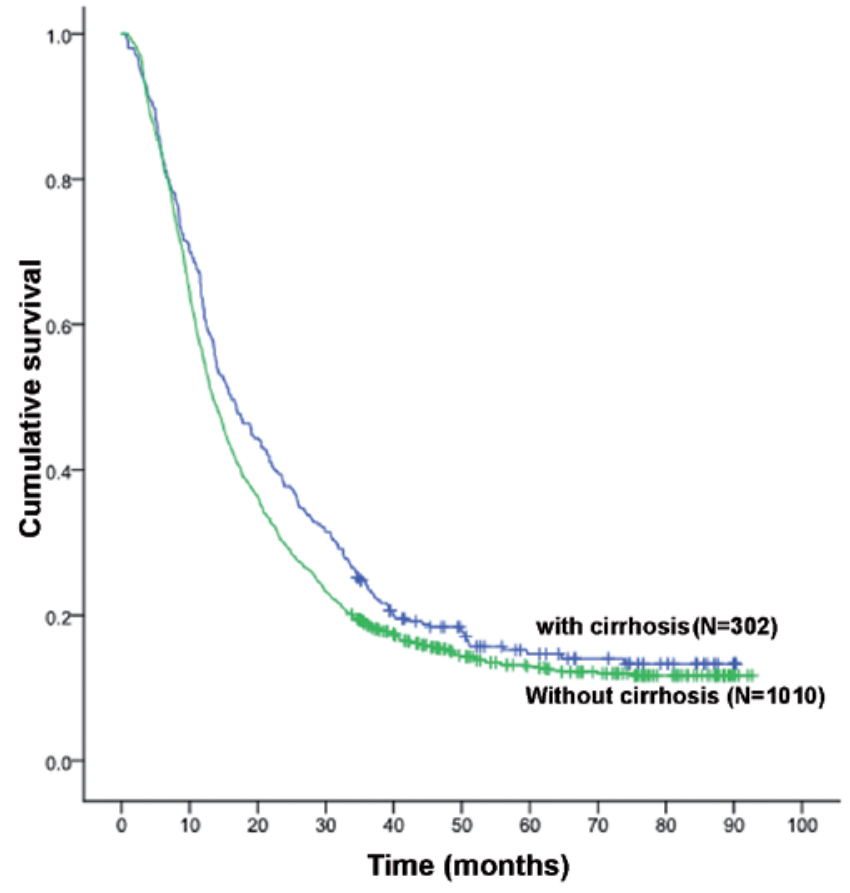

Figure 1. Overall survival (OS) in intrahepatic cholangiocarcinoma patients with and without cirrhosis: The 1-, 3- and 5-year OS rated for patients with cirrhosis were $62.3,24.1$ and $14.7 \%$, respectively, which were significantly higher compared with the corresponding rates in patients without cirrhosis (55.4, 18.7 and $13.0 \%$, respectively), with median survival times of 16.0 vs. 13.0 months, respectively $(\mathrm{P}<0.038)$.

countries (15). Cirrhosis is most likely associated with HBV infection in China (26), and $90.1 \%$ of cases with cirrhosis in the present series were seropositive for hepatitis B surface antigen, indicating HBV-related cirrhosis. Although the prevalence in our study may not prove a causal association between cirrhosis and ICC, as is the case with HCC, it indicates a correlation between the two. Patients with cirrhosis have a $~ 16$-fold higher risk of HCC compared with inactive carriers (8). Therefore, future investigation should examine whether cirrhosis plays a synergistic role in ICC development in patients with HBV infection.

It is generally hypothesized that the prognosis of ICC is worse compared with that of HCC following surgical treatment $(2,5,27)$. Complete surgical resection is the only curative treatment for ICC; however, similar to previous reports $(2,6,7,25)$, the OS of this entire cohort indicated that the prognosis of ICC is dismal following surgical management, with an MST of only 14.0 months, as the disease is usually advanced at the time of diagnosis. There are several known factors affecting the prognosis of ICC after surgery, including surgical margin status, multiple tumors, LN metastasis and vascular invasion $(2,6,7,25,28)$. In earlier studies on ICC, cirrhosis (21), unlike HBV infection $(13,18,19)$, was shown to be an independent unfavorable prognostic factor for survival, but little is known on the underlying mechanism. However, the multivariate analysis in this series and our former study (20) revealed that cirrhosis was an independent favorable prognostic factor for survival of ICC patients following surgery. Different sample sizes may be the reason for the different results reported by these studies regarding the role of cirrhosis in ICC prognosis.

In the present study, all the patients with underlying liver disease had well-compensated liver function (Child A), which, in non-cirrhotic cases, should not significantly affect the extent of hepatectomy or postoperative morbidity and mortality. However, as reported by an earlier study on ICC (21), cirrhosis exerts a negative effect on major hepatectomy, and cirrhotic patients in our study tended to have a smaller resection range (Table III). Non-cirrhotic patients may be more amenable to resection due to a relatively better preserved liver function, as in HCC tumors $(16,17)$. However, the R0 resection rate was significantly higher among cirrhotic patients $(\mathrm{P}<0.001)$, while the rates of non-curative resection $[\mathrm{R} 1(\mathrm{P}=0.002)$ and $\mathrm{R} 2(\mathrm{P}=0.027)]$ and exploratory laparotomy $(\mathrm{P}=0.045)$ were significantly higher among non-cirrhotic patients, which may be one of the reasons for the superior survival of ICC patients with cirrhosis. ICCs in non-cirrhotic patients tended to be larger, with a lower incidence of capsule formation, and at a more advanced stage at diagnosis compared with those in cirrhotic patients (Table II), which may be the reason for the better surgical margin status and survival in ICC patients with cirrhosis. ICC patients with cirrhosis exhibited a significantly better survival compared with those without cirrhosis (Fig. 1), which may not be attributed to early tumor detection. Although our data demonstrated that patients with cirrhosis had significantly smaller tumors compared with those without cirrhosis (Table I), tumor size was not found to be an independent prognostic factor for ICC patients. In the 
present study, the presence of cholelithiasis, HBV infection, elevated CA19-9 and CEA levels, surgical margin status and certain pathological characteristics, such as multiple tumors, capsule formation, lymphatic metastasis and vascular invasion, were significantly associated with the presence of cirrhosis, but the associations were not causative, and multivariate analysis demonstrated that these factors together with cirrhosis were all independent prognostic factors for ICC (Table IV). The differences in the clinicopathological characteristics between ICC patients with and those without cirrhosis may be due to different underlying pathogenic mechanisms in the two groups of patients.

According to previous studies, the development of HCC in cirrhotic and non-cirrhotic livers may be underlined by distinct mechanisms $(8,9)$, which has not been proven in ICC patients. In the present study, in the clinical setting, ICC patients with cirrhosis exhibited different and unique characteristics compared with patients without cirrhosis. The findings of this study suggested that ICC associated with cirrhosis may display a biological behavior similar to that of $\mathrm{HCC}$ and, thus, have a better prognosis. Although the etiology of ICC remains unclear, there is growing evidence suggesting that ICC associated with cirrhosis may be derived from the same hepatic progenitor cells as $\operatorname{HCC}(13,14,18,19)$ and, thus, behaves more like HCC, which is generally considered to have a more favorable prognosis compared with $\operatorname{ICC}(2,5,27,29)$. The observations of the present study indicate that ICC occurring in patients with cirrhosis may share a common carcinogenic process with HCC. Compared with non-cirrhotic patients, cirrhotic ICC patients were more likely younger and male, a profile resembling that of HCC patients $(18,19)$. The formation of vascular tumor thrombi, one of pathological characteristics of HCC, was observed more often among ICC patients with cirrhosis compared with those without cirrhosis. In contrast to a previous study (21), LN metastasis and perineural invasion, which are typical pathological characteristics of adenocarcinoma, were less often found in ICC patients with cirrhosis compared with those without cirrhosis. AFP is often used as a tumor marker for HCC and, in the present study, a significantly higher number of cirrhotic ICC patients exhibited elevated serum AFP levels compared with non-cirrhotic patients, suggesting that the ICC cells may exhibit hepatocellular differentiation. These findings also suggest that ICC with cirrhosis and HCC may share a common carcinogenic process.

The present study had several limitations. First, a small number of patients with mild fibrosis or steatosis were included, which may have affected the findings; however, none of these patients had true cirrhosis and, therefore, were considered eligible for inclusion in the cohort of non-cirrhotic patients. Patients with HCV infection, a known inciting factor of hepatocarcinogenesis (12), were not included in the present study due to the small case series of $\mathrm{HCV}$ infection. A number of patients received non-radical resection and a considerable percentage of non-anatomical hepatectomies were included in this study, due to the advanced tumor stage at the time of diagnosis and the high incidence of chronic liver disease, such as HBV infection and cirrhosis, prevalent in China. Furthermore, although it included the largest case series of ICC patients, this study was retrospective in nature, which may be associated with certain limitations with regards to data selection.
In conclusion, cirrhosis is an independent favorable prognostic factor for survival of ICC patients, due to the distinct biological characteristics as well as the different pathogenic mechanism in this subgroup of patients. More emphasis should be placed on aggressive surgical treatment for ICC patients with cirrhosis, considering safety and better survival in this group. Non-cirrhotic patients may lack the typical 'field-defect' of a cirrhotic liver; however, these patients may harbor a molecular field defect that differs from that of a cirrhotic liver, leading to higher-risk pathological characteristics, lower resection rates and worse survival. Further investigation should be focused on the genomic profile of livers with and without cirrhosis in order to elucidate the different pathogenic mechanisms underlying the development of ICC, in order to design novel targeted treatments to improve the survival of ICC patients.

\section{References}

1. Sempoux C, Jibara G, Ward SC, Fan C, Qin L, Roayaie S, Fiel MI, Schwartz M and Thung SN: Intrahepatic cholangiocarcinoma: New insights in pathology. Semin Liver Dis 31: 49-60, 2011.

2. Endo I, Gonen M, Yopp AC, Dalal KM, Zhou Q, Klimstra D, D'Angelica M, DeMatteo RP, Fong Y, Schwartz L, et al: Intrahepatic cholangiocarcinoma: Rising frequency, improved survival, and determinants of outcome after resection. Ann Surg 248: 84-96, 2008.

3. Khan SA, Taylor-Robinson SD, Toledano MB, Beck A, Elliott P and Thomas HC: Changing international trends in mortality rates for liver, biliary and pancreatic tumours. J Hepatol 37: 806-813, 2002 .

4. Nakanuma Y, Sasaki M, Ikeda H, Sato Y, Zen Y, Kosaka K and Harada K: Pathology of peripheral intrahepatic cholangiocarcinoma with reference to tumorigenesis. Hepatol Res 38: 325-334, 2008.

5. The general rules for the clinical and pathological study of primary liver cancer. Liver cancer study group of Japan. Jpn J Surg 19: 98-129, 1989.

6. Ohtsuka M, Ito H, Kimura F, Shimizu H, Togawa A, Yoshidome H and Miyazaki M: Results of surgical treatment for intrahepatic cholangiocarcinoma and clinicopathological factors influencing survival. Br J Surg 89: 1525-1531, 2002.

7. Wu ZF, Zhang HB, Yang N, Zhao WC, Fu Y and Yang GS: Postoperative adjuvant transcatheter arterial chemoembolisation improves survival of intrahepatic cholangiocarcinoma patients with poor prognostic factors: Results of a large monocentric series. Eur J Surg Oncol 38: 602-610, 2012.

8. Fattovich G, Stroffolini T, Zagni I and Donato F: Hepatocellular carcinoma in cirrhosis: Incidence and risk factors. Gastroenterology 127: S35-S50, 2004.

9. Tretiakova MS, Shabani-Rad MT, Guggisberg K, Hart J, Anders RA and Gao ZH: Genomic and immunophenotypical differences between hepatocellular carcinoma with and without cirrhosis. Histopathology 56: 683-693, 2010.

10. Paquet KJ, Gad HA, Lazar A, Koussouris P, Mercado MA, Heine WD, Jachman-Jahn V and Ruppert W: Analysis of factors affecting outcomes after hepatectomy of patients with liver cirrhosis and small hepatocellular carcinoma. Eur J Surg 164: 513-519, 1998.

11. Bréchot C: Pathogenesis of hepatitis B virus-related hepatocellular carcinoma: Old and new paradigms. Gastroenterology 127 (5 Suppl 1): S56-S61, 2004

12. Perumal V, Wang J, Thuluvath $\mathrm{P}$, Choti $\mathrm{M}$ and Torbenson $\mathrm{M}$ : Hepatitis $\mathrm{C}$ and hepatitis $\mathrm{B}$ nucleic acids are present in intrahepatic cholangiocarcinomas from the United States. Hum Pathol 37: 1211-1216, 2006.

13. Lee CH, Chang CJ, Lin YJ, Yeh CN, Chen MF and Hsieh SY: Viral hepatitis-associated intrahepatic cholangiocarcinoma shares common disease processes with hepatocellular carcinoma. Br J Cancer 100: 1765-1770, 2009.

14. Palmer WC and Patel T: Are common factors involved in the pathogenesis of primary liver cancers? A meta-analysis of risk factors for intrahepatic cholangiocarcinoma. J Hepatol 57: 69-76, 2012.

15. Shaib YH, El-Serag HB, Davila JA, Morgan R and McGlynn KA: Risk factors of intrahepatic cholangiocarcinoma in the United States: A case-control study. Gastroenterology 128: 620-626, 2005. 
16. Pandey D, Lee KH, Wai CT, Wagholikar G and Tan KC: Long term outcome and prognostic factors for large hepatocellular carcinoma (10 cm or more) after surgical resection. Ann Surg Oncol 14: 2817-2823, 2007.

17. Poon RT, Fan ST, Lo CM, Liu CL, Ng IO and Wong J: Long-term prognosis after resection of hepatocellular carcinoma associated with hepatitis B-related cirrhosis. J Clin Oncol 18: 1094-1101, 2000.

18. Peng NF, Li LQ, Qin X, Guo Y, Peng T, Xiao KY, Chen XG, Yang YF, Su ZX, Chen B, et al: Evaluation of risk factors and clinicopathologic features for intrahepatic cholangiocarcinoma in Southern China: A possible role of hepatitis B virus. Ann Surg Oncol 18: 1258-1266, 2011

19. Zhang L, Cai JQ, Zhao JJ, Bi XY, Tan XG, Yan T, Li C and Zhao P: Impact of hepatitis B virus infection on outcome following resection for intrahepatic cholangiocarcinoma. J Surg Oncol 101: 233-238, 2010.

20. Luo X, Yuan L, Wang Y, Ge R, Sun Y and Wei G: Survival outcomes and prognostic factors of surgical therapy for all potentially resectable intrahepatic cholangiocarcinoma: A large single-center cohort study. J Gastrointest Surg 18: 562-572, 2014.

21. Li YY,Li H, Lv P, Liu G, Li XR, Tian BN and Chen DJ: Prognostic value of cirrhosis for intrahepatic cholangiocarcinoma after surgical treatment. J Gastrointest Surg 15: 608-613, 2011.

22. Scheuer PJ: Classification of chronic viral hepatitis: A need for reassessment. J Hepatol 13: 372-374, 1991.

23. Strasberg SM: Nomenclature of hepatic anatomy and resections: A review of the Brisbane 2000 system. J Hepatobiliary Pancreat Surg 12: 351-355, 2005.
24. Edge SB and Compton CC: The American Joint Committee on Cancer: The 7th edition of the AJCC cancer staging manual and the future of TNM. Ann Surg Oncol 17: 1471-1474, 2010.

25. Farges O, Fuks D, Boleslawski E, Le Treut YP, Castaing D, Laurent A, Ducerf C, Rivoire M, Bachellier P, Chiche L, et al: Influence of surgical margins on outcome in patients with intrahepatic cholangiocarcinoma: A multicenter study by the AFC-IHCC-2009 study group. Ann Surg 254: 824-830, 2011

26. Hou J, Liu Z and Gu F: Epidemiology and prevention of hepatitis $B$ virus infection. Int J Med Sci 2: 50-57, 2005.

27. Zhou XD, Tang ZY, Fan J, Zhou J, Wu ZQ, Qin LX, Ma ZC, Sun HC, Qiu SJ, Yu Y, et al: Intrahepatic cholangiocarcinoma: Report of 272 patients compared with 5,829 patients with hepatocellular carcinoma. J Cancer Res Clin Oncol 135: 1073-1080, 2009.

28. Wang Y, Li J, Xia Y, Gong R, Wang K, Yan Z, Wan X, Liu G, Wu D, Shi L, et al: Prognostic nomogram for intrahepatic cholangiocarcinoma after partial hepatectomy. J Clin Oncol 31: 1188-1195, 2013

29. Xu J, Igarashi S, Sasaki M, Matsubara T, Yoneda N, Kozaka K, Ikeda H, Kim J, Yu E, Matsui O and Nakanuma Y: Intrahepatic cholangiocarcinomas in cirrhosis are hypervascular in comparison with those in normal livers. Liver Int 32: 1156-1164, 2012. 